\title{
Gibt es einen Kodex für die Grammatik des Neuhochdeutschen und, wenn ja, wie viele? Oder: Ein Plädoyer für Sprachkodexforschung*
}

\begin{abstract}
Sprachverfall wird in der öffentlichen Sprachdiskussion nicht selten mit der Unkenntnis oder Missachtung von sprachlichen Regeln in Verbindung gebracht. Als Instanzen, wo sich (explizite) Sprachregeln gesellschaftlich relevant verkörpern, können Sprachkodizes gesehen werden. Vor diesem Hintergrund wird im Text der Begriff des Sprachkodex in verschiedenen Dimensionen präzisiert und eine Subklassifikation in Kern- und Parakodex vorgeschlagen. Dem folgt ein Plädoyer für eine Sprachkodexforschung, in der die traditionell eher marginalen Perspektiven auf Sprachkodizes zu erweitern und zu systematisieren sind.
\end{abstract}

\section{Einführendes}

Die Rede vom Sprachverfall wurde von der Sprachwissenschaft aus guten Gründen schon oft kritisiert, zumindest relativiert und historisch situiert (z.B. Beck 1996; Bittner/Köpcke 2008; Görtz 1990; Keller 2006; Klein 1986; Maercker 2006). Für die öffentliche Sprachdiskussion, die an diesem Punkt mindestens seit dem Ende des 19. Jahrhunderts mit der literarischen Sprachreflexion verknüpft ist (Görtz 1990), besaß das bisher kaum große Nachwirkungen. Dort verkörpert sich die Rede vom Sprachverfall weiterhin in recht unterschiedlichen Formen. Eine Variante sieht so aus, dass der Sprachverfall mit der angeblichen Missachtung von Regeln in Verbindung gebracht wird. Man greift „Fehler" im Sprachgebrauch auf und sieht darin die Unkenntnis gültiger Sprachnormen. „Falscher" Sprachgebrauch zeigt demnach den allmählichen Untergang des Deutschen, zumindest des, guten", „normgerechten“ Deutsch. Regelunkenntnis führt, so die Unterstellung, zu Sprachverfall, da wesentliche Sprachgesetze nicht mehr befolgt werden.

In dieser Sichtweise könnten öffentliche Sprachdiskussion und sprachwissenschaftliche Forschung bis zu einem gewissen Punkt zueinander finden. Denn auch für die Sprachwissenschaft ist das Kalkulieren mit Regeln von großer Bedeutung: Wer eine Sprache systematisch beschreiben und analysieren möchte, wird in der einen oder anderen Form mit einem Regel-

Ich danke Albrecht Plewnia, dessen wertvolle Hinweise und Kommentare zum Manuskript mir bei der Endredaltion dieses Textes sehr geholfen haben. 
begriff arbeiten müssen. Noch diesseits aller grammatiktheoretischen Differenzen lässt sich davon ausgehen, dass jedes Sprachsystem im Kern aus einer begrenzten Anzahl von Sprachregeln besteht.

Nun unterscheiden sich allerdings die nicht-sprachwissenschaftliche und die sprachwissenschaftliche Sicht auf Sprachregeln in einem entscheidenden Punkt: Linguistische Laien sehen in sprachlichen Regeln meistens explizite Regelformulierungen, wie man sie etwa aus dem Schulunterricht kennt. Solche (präskriptiven) Sprachregeln sollen dann etwa in den Duden-Publikationen und verwandten Sprachanweisungen stehen. Sprachwissenschaftler dagegen arbeiten mit einem deskriptiven Regelbegriff, der gerade nicht an expliziten Formulierungen, sondern an der ,,unbewussten ${ }^{\text {"c Wirksamkeit }}$ von Regeln ansetzt. Wer eine Sprache spricht, folgt diesen (,,subsistenten“, „impliziten“) Regeln, auch wenn er nicht in der Lage ist, sie ausdrücklich anzugeben. Demgegenüber stellen explizit formulierte Regeln, die oft mit dem Begriff „Sprachnormen“" gleichgesetzt werden, für Sprachwissenschaftler - wenn überhaupt - nur einen sekundären Gegenstand dar. Insbesondere zielt die Sprachwissenschaft auch nicht darauf ab, explizite Sprachnormen präskriptiv zu verhängen. Ihre Tätigkeit ist vielmehr der deskriptiven Rekonstruktion von Sprachgebrauchsregeln gewidmet, nicht der Durchsetzung von Regeln in einer Sprachgemeinschaft.

Vor dieser Spannung zwischen der sprachwissenschaftlichen und der nicht-sprachwissenschaftlichen Sicht auf „,Regeln“ möchte ich mich hier mit der Frage der Kodifizierung des Deutschen beschäftigen. Dieses Thema berührt einerseits die nicht-sprachwissenschaftliche Sicht auf Regeln, weil sich die Kodifizierung einer Sprache in expliziten Regelformulierungen ausdrückt. Andererseits ist die Kodifizierungsproblematik ein Gegenstand, der in der bisherigen Forschung zwar immer wieder berührt wurde, der aber noch nie zum primären Forschungsobjekt erhoben wurde. Darin sehe ich ein großes Defizit. Diese Skizze stellt daher auch ein Plädoyer zur Etablierung einer Sprachkodexforschung dar. Im Laufe des Texts soll sich insofern zeigen, dass sowohl die öffentliche Sprachdiskussion als auch die sprachwissenschaftliche Forschung erheblich gewinnen könnte, wenn man genauer als bisher über die Geschichte, das Ausmaß und die Konsequenzen der Kodifizierung des Deutschen Bescheid wüsste.

\section{Was ist ein Sprachkodex?}

Mit dem Begriff der Kodifizierung bezeichnet man den Umstand, dass eine bestimmte Form einer Sprache ausdrücklich in schriftlichen Regelwerken beschrieben bzw. normiert wird. In diesen metasprachlich einschlägigen Schriftstücken (v.a. Grammatiken, Wörterbücher) liegt der Kodex einer Sprache vor, der sich auf eine funktional besonders leistungsfähige und prestigebehaftete Varietät einer Sprache („,Standardvarietät ${ }^{\circ}$ ) bezieht (z.B. Metzler 
Lexikon Sprache 2010, Artikel Kodifizierung). Neben gebrauchsorientierten Zugängen zur Standardsprache gibt es üblicherweise kodexorientierte Versuche festzustellen, was zur deutschen Standardvarietät gehört und was nicht (Klein 2013, S. $26 \mathrm{ff}$.).

In sprachhistorischer Perspektive lässt sich davon ausgehen, dass durch die Existenz eines Sprachkodex die Geschichte einer Sprache ein neues Entwicklungsstadium erreicht. Dies legen jedenfalls die einschlägigen Übersichtswerke nahe, wenn sie die Geschichte der Grammatikschreibung und der Lexikografie in eine chronologische Darstellung des Deutschen integrieren und damit der Kodifizierung, zumindest mittelbar, eine besondere Wirkungskraft zuschreiben (z.B. Schmidt 2007, Kap. 1.5.3, 1.6.2, 1.7.5, 1.8.4; Polenz 1994, Kap. 5.6/5.7; ders. 1999, Kap. 6.6). In der Kodifizierung einer Sprache wird demnach ein wichtiger Aspekt der Standardisierung einer Sprache, d.h. der Herausbildung einer Standardvarietät, gesehen (Ammon 1986, 1995, S. 74 ff., 2005).

Ganz allgemein kann man vor diesem Hintergrund festhalten, dass sich Sprachen angesichts der Frage prinzipiell unterscheiden, ob sie kodifiziert sind oder nicht. Für die großen europäischen Kultursprachen (Französisch, Spanisch, Italienisch, Englisch, Deutsch) existiert eine teilweise weit in die Vergangenheit zurückreichende Kodifizierungsgeschichte, während andere Sprachen überhaupt nicht oder - sprachhistorisch gesehen - erst seit kurzem kodifiziert vorliegen. Eine kleine Analogie zur Rechtssphäre mag die Auswirkungen dieser Kodifizierungen illustrieren: Die Ordnung einer Gesellschaft ändert sich in dem Moment grundlegend, wenn gültige Gesetze und Verbote nicht mehr nur mündlich aktualisiert, sondern schriftlich festgehalten werden. Auf einer Gesetzesstele wurden grundlegende Regeln eben ,in Stein gemeißelt ${ }^{c c}$.

In alteuropäischer Tradition lässt sich hier auf die Bedeutung der mesopotamischen Gesetzgebung (Codex Hammurabi), der mosaischen Gesetzestafeln (Zehn Gebote) und der einschlägigen juristischen Rechtskodifikationen (Codex Iustinianus) verweisen. In derlei normativ-schriftlichen Gründungsakten verkörpert sich das Bewusstsein, dass Regeln dann eine größere Legitimität gewinnen, wenn sie schriftlich formuliert sind. Auf Sprache übertragen: Wer in einer Situation kommuniziert, in der bestimmte sprachliche Einheiten ausdrücklich gegenüber anderen favorisiert werden, agiert unter anderen Rahmenbedingungen als derjenige, dem entsprechende Schriften nicht zur Verfügung stehen. Oder anders perspektiviert: Wenn es einen Sprachkodex gibt, wird das individuelle und kollektive Sprachbewusstsein samt der korrespondierenden Sprachaufmerksamkeit anders aussehen als in einer Situation, wo keine Sprachregeln „festgeschrieben " sind. Schon vor diesem Hintergrund dürfte deutlich werden, dass die Sprachkodexforschung keinesfalls an den Rand, sondern ins Zentrum einer wirklichkeitsnahen Sprachwissenschaftskonzeption gehört. 
Nun sollte man die Analogie zwischen der Sphäre des Rechts und der Sphäre der Sprache sicher nicht zu weit treiben. Sie kann auch in die Irre führen. Eine Anknüpfung an dieses schriftorientierte Legitimitätsdenken scheint mir aber immer dann vorzuliegen, wenn mit mehr oder weniger großer Geste auf die Einschlägigkeit von Sprachkodifizierungen hingewiesen wird. Dies kann sowohl in der öffentlichen Sprachdiskussion erfolgen als auch in der sprachwissenschaftlichen Forschung. In beiden Fällen sieht man in Kodifizierungen relevante Instanzen unserer Sprachwirklichkeit. Ein erheblicher Unterschied liegt hier allerdings wieder in der Perspektive und dem Deutungshorizont: Nicht-Sprachwissenschaftler beziehen sich bekanntlich oft affirmativ auf die (angeblichen, zeitlos ,gültigen ") Kodifizierungen, etwa in den Duden-Publikationen. Für Sprachwissenschaftler sind die Kodifizierungen dagegen eher gegebene, oft nicht besonders relevante Instanzen der Standardisierung, deren präskriptive Kraft von Fall zu Fall relativiert und kritisiert werden muss. Für beide gilt: Wer Sprachkodifizierungen in den Blick nimmt, sollte auch im Detail darüber Rechenschaft ablegen können, von welchem Kodex-Begriff er ausgeht und welche Gehalte damit verbunden sind. Gefragt ist also eine Präzisierung von Sprachkodex, die durch eine realistische Sicht auf unsere Sprachwirklichkeit gestützt wird. Vor diesem Hintergrund schlage ich die folgende Definition vor:

Zum Sprachkodex einer Sprache gehören alle metasprachlichen Schriften, die für eine Sprachgemeinschaft zu einem bestimmten Zeitpunkt als Normautoritäten zur Verfügung stehen und von ihr auch als Normautoritäten wahrgenommen werden. Der Sprachkodex kann sich auf unterschiedliche Ebenen und Instanzen der Sprache beziehen: Aussprache, Schreibung, Grammatik (Wort- und Wortformenbildung, Syntax), Lexik, Semantik, Pragmatik.

Mit dieser Definition knüpfe ich an den üblichen Gebrauch dieses Terminus in der Sprachwissenschaft und seine fachlexikografische Beschreibung an. Einiges sei freilich ausdrücklich erläutert und zugespitzt: Nach meiner Definition kann man davon ausgehen, dass ein Sprachkodex in der Regel nicht aus einer einzigen Schrift, sondern aus mehreren, ggf. (sehr) vielen Texten besteht. Das richtet sich auch gegen die häufig zu beobachtende Unterstellung, dass letztlich nur eine bestimmte Schrift als Kodex zu begreifen ist. Was für die Orthographie in bestimmten historischen Situationen noch eine gewisse Plausibilität haben mag, nämlich die Identifikation eines Kodex mit einem einzigen rechtsförmigen Text, das ist auf den anderen Sprachebenen und je nach historischer Situation eine suggestive Annahme, die wenig mit der Sprachrealität zu tun hat. Denn Grammatik und Lexik, Textstrukturen und Sprechaktvollzug wurden für die deutsche Sprache bekanntlich noch nie ,staatlich geregelt". Es gibt keine Bibel der Grammatik 
oder der Syntax, so wie es - wenigstens in einer bestimmten Sicht - eine Bibel der Rechtschreibung gibt.

In den meisten Fällen besteht der Kodex also aus einer Reihe von Schriften, die von der Sprachgemeinschaft in Normfragen von Fall zu Fall als Normautoritäten wahrgenommen und konsultiert werden. Letzteres richtet sich vor allem dagegen, tendenziell jede - auch jede wissenschaftliche Sprachbeschreibung als Kodifikation zu verstehen. Für die Aufnahme in den Kodex sind nämlich die faktische Reichweite und der tatsächliche Gebrauch innerhalb einer Sprachgemeinschaft von entscheidender Bedeutung. Es muss also Hinweise darauf geben, dass eine bestimmte, der Sprache gewidmete Schrift in orientierender Absicht von vielen Menschen benutzt wird. Das geschieht wahrscheinlich oft in sprachlichen Zweifelsfällen (dazu näher Klein 2003, 2006, 2009, 2011).

Natürlich ist es nicht einfach, die breite Nutzung einer Schrift tatsächlich nachzuweisen. Wie so oft stellen sich in der Folge von Definitionen gravierende empirisch-methodologische Probleme. Sie können sicher nicht immer definitiv gelöst werden, vor allem nicht für die (fernere) Vergangenheit. Deshalb sei hier nur angedeutet, dass man an dieser Stelle etwa auf die wiederholte Auflage von Büchern und ihre Resonanz in der öffentlichen Diskussion verweisen kann, wenn man die Zusammenstellung des Kodex erwägt. Bestimmte Publikationen gehören im Buchhandel faktisch zu den Bestsellern. Ihre Autoren tauchen in der medialen Sprachdiskussion immer wieder auf. Für linguistische Fachpublikationen i.e.S. dürfte eine solche Konstellation in der Regel nicht vorliegen. Die meisten Grammatiken des Deutschen sind zwar beeindruckende Bücher, werden aber - das lässt sich ohne weitere Untersuchung feststellen - weder von linguistischen Laien noch in der öffentlichen Sprachdiskussion wahrgenommen. Sie gehören daher nicht zum Kodex.

Angesichts dieser Problematik kommt schon eine erste, durchaus zentrale Aufgabe der Sprachkodexforschung in Sichtweite: Welche Texte gehören überhaupt zu einem gegebenen Zeitpunkt zum Sprachkodex? Jede Antwort auf diese Frage steht unter dem Druck, das definitorische Merkmal der ,breiten Resonanz in einer Sprachgemeinschaft ${ }^{`}$ so gut wie nach den jeweiligen Rahmenbedingungen eben möglich empirisch aufzugreifen und einzulösen. Bei manchen Schriften wird es dann vermutlich offen bleiben, ob sie tatsächlich zum zeitgenössischen Sprachkodex gerechnet werden können oder nicht. Bei anderen hingegen dürfte eine Zuordnung auf der Basis der obigen Definition einigermaßen unumstritten sein.

Angesichts des Umstands, dass oben genannte Definition potenziell recht viele Texte zum Kodex einer Sprache rechnet, möchte ich darüber hinaus noch eine Subklassifizierung innerhalb der Kodexschriften vorschlagen. Definitorisch sei daher der Kernkodex vom Parakodex unterschieden: 
Zum Kernkodex einer Sprache gehören alle Kodextexte, die primär für formelle Gebrauchssituationen (z.B. Schule, Verwaltung) verfügt werden und die direkt oder indirekt offiziell ( $g g f$. „staatlich c) legitimiert sein können, z.B. „Amtliche Regelung ${ }^{c 6}$ der deutschen Orthographie, Schulgrammatiken (inkl. Lehrplänen). Zum Parakodex gehören alle Kodextexte, die nicht Bestandteil des Kernkodex sind.

Die Differenzierung zwischen Kern- und Parakodex wirft hauptsächlich auf der Seite des Parakodex die Frage auf, welche Schriften dort involviert sein könnten. Der Kernkodex steht demgegenüber dem traditionellen Sprachkodexbegriff sehr nahe. Er wird daher relativ unproblematisch sein. Illustrierend möchte ich daher feststellen, dass zum Parakodex der (späten) neuhochdeutschen Grammatik beispielsweise die folgenden Orientierungswerke aus der zweiten Hälfte des 19. und der ersten Hälfte des 20. Jahrhunderts gehören sollten:

Sanders, Daniel (1872): Kurzgefasstes Wörterbuch der Hauptschwierigkeiten in der deutschen Sprache. Berlin.

Brasch, Martin Wilhelm (1874): Die deutsche Grammatik und ihre Schwierigkeiten. Stuttgart.

Grube, August W. (1876): Streiflichter auf die Wandlungen und Schwankungen im neuhochdeutschen Sprachgebrauch. Leipzig.

Lehmann, August (1877): Sprachliche Sünden der Gegenwart. Braunschweig.

Keller, Karl Gottlieb (1879): Deutscher Antibarbarus. Beiträge zur Förderung des richtigen Gebrauchs der Muttersprache. Stuttgart.

Andresen, Karl Gustav (1880): Sprachgebrauch und Sprachrichtigkeit im Deutschen. Heilbronn.

Bruns, Wilhelm (1880): Ratgeber in den Schwierigkeiten der deutschen Sprache. Düsseldorf.

Wustmann, Gustav (1891): Allerhand Sprachdummheiten. Kleine deutsche Grammatik des Zweifelhaften, des Falschen und des Häßlichen. Leipzig.

Matthias, Theodor (1892): Sprachleben und Sprachschäden. Ein Führer durch die Schwankungen und Schwierigkeiten des deutschen Sprachgebrauchs. Leipzig.

Bennewitz, Alexander (1898): Die Schwierigkeiten unserer Muttersprache. Übersichtliche Zusammenstellung der zweifelhaften Fälle im mündlichen und schriftlichen Sprachgebrauche, mit besonderer Berücksichtigung der kaufmännischen Sprache. Leipzig.

Engel, Eduard (1922): Gutes Deutsch. Ein Führer durch Falsch und Richtig. Leipzig.

Schneider, Karl (1931): Was ist Gutes Deutsch? Ein Führer durch die Schwierigkeiten und Zweifelsfälle des heutigen deutschen Sprachgebrauchs. 2., verm. Aufl. München. 
Für alle diese Schriften lässt sich annehmen, dass sie keine fachlinguistischen Spezialpublikationen darstellen. Sie waren oft zur Orientierung in sprachlichen Zweifelsfällen („Normierung ${ }^{\circ}$ ) an die sprachinteressierte Öffentlichkeit gerichtet und wurden - das kann man zumindest mittelbar aus den oft zahlreichen Auflagen schließen - auch rege rezipiert. Für die Gegenwart wären auf dieser Linie etwa die einschlägigen Publikationen von W. Schneider, E. Ch. Hirsch und B. Sick zum Parakodex zu rechnen.

Was die Unterscheidung von Kern- und Parakodex angeht, so sei abschließend festgehalten, dass sie zwar einen dichotomen Eindruck erweckt, faktisch aber auch mit Skalen und Übergangszonen kalkuliert werden muss. Manche Schriften werden die Definitionskriterien für den Kern- oder den Parakodex vielleicht nur in Teilen erfüllen. Andere dagegen können ohne große Diskussion dem einen oder anderen Kodexteil zugeordnet werden. Es ist also mit mehr oder weniger typischen Instanzen des Kern- bzw. Parakodex zu rechnen. Dazu kommen, genauso wie bei der vorgelagerten Kodex-Definition, die üblichen empirischen Unwägbarkeiten. Nicht immer lassen sich aufgrund des historischen Kontexts die Definitionskriterien erschöpfend überprüfen. Auch hier gilt also, dass mit einer Begriffsbestimmung wieder empirische Probleme aufgeworfen werden, die nicht überall gelöst werden können. Durch empirische Defizite wird freilich der Sinn und Zweck einer gegebenen Definition nicht grundsätzlich geschmälert.

\section{Sprachkodexforschung: eine Skizze}

Mit den obigen Klärungen wurde der primäre Gegenstand der Sprachkodexforschung definiert: Es handelt sich um eine Reihe von Texten (= Sprachkodex), die für das kommunikative Orientierungsbewusstsein einer Sprachgemeinschaft eine maßgebliche Rolle spielen. Im Sprachkodex manifestiert sich der institutionell gefestigte Teil des kollektiven Sprachbewusstsein einer Zeit. Es wird in schriftlich-formalen Texten mit konstitutiv metasprachlichem Charakter greifbar, insbesondere insofern Sprache mit Wertungen und sozialsymbolischen Gehalten aufgeladen wird. Was schriftlich kodifiziert wurde, ist bis zu einem gewissen Grad auch in den Köpfen der Sprecher präsent. Man kann darüber hinaus ohne große Spekulation annehmen, dass der Sprachkodex nicht nur metasprachlich, sondern auch objektsprachlich relevant ist. Er wird den realen Sprachgebrauch entweder in Teilen spiegeln oder als präskriptive Folie anleiten. An welchen Punkten genau und in welchem Ausmaß es zu einer solchen Wirkung gekommen ist, kann als eine der Hauptaufgaben der Sprachkodexforschung verstanden werden.

Aufbauend auf obigen Vorklärungen sollen nun einige Forschungshorizonte der Sprachkodexforschung vorgestellt werden. Wenn ermittelt wurde, welche Texte zum Kodex einer Zeit gehören, können diese Schrif- 
ten nämlich unter verschiedenen Aspekten näher analysiert werden. Den Forschungsentwurf, der sich daraus ergibt, möchte ich zugleich mit Hinweisen auf bisherige Arbeiten zur Kodifizierungsproblematik verbinden. Es soll zumindest angedeutet werden, in welchen Problemkonstellationen der Sprachkodex des Deutschen bisher bereits in den Blick der linguistischen Forschung geraten ist.

Generell lässt sich festhalten, dass die Kodifizierung des Deutschen bisher meistens nur am Rande anders akzentuierter Arbeiten berührt wurde. ${ }^{1}$ Die Thematisierung von Kodizes war meistens ein Randphänomen. Insbesondere als heuristische Mittel für Arbeiten zu gegenwärtigen oder historischen Sprachformen kamen sie gelegentlich in den Blick (z.B. Voeste 1999, Kap. 3; Dovalil 2006, bes. Kap. 3; Elspaß 2005; Hennig (Hg; 2009). Ähnliches gilt für die mehr oder weniger implizite Thematisierung von Kodifizierungen, wenn sprachbezogene Nachschlagewerke im Beratungskontext eine Rolle spielen (z.B. Riegel 2007). Je weiter man in die Vergangenheit zurückgeht, desto mehr können wissenschaftshistorische Arbeiten - zumindest in Teilen - auch als Beiträge zur historischen Sprachkodexforschung gelten (z.B. Hundt 2000). Der Kodifizierung an und für sich waren hingegen bisher nur wenige Projekte gewidmet. In der Sprachkodexforschung wird die Kodifizierung einer Sprache nämlich in den Mittelpunkt gestellt, nicht am Rande mitbehandelt oder zum Ausgangspunkt von Untersuchungen mit anderer Stoßrichtung gemacht.

Ferner gilt, dass kodexbezogen, wenn überhaupt, vor allem die Aussprache (z.B. Hollmach 2007; Ehrlich 2008) und die Schreibung (z.B. Höchli 1981; Schlaefer 1980, 1981; Schmidt-Wilpert/Lappé 1981; Stanze 1994; Takada 1997; Takahashi 1996) behandelt wurden. In beiden Fällen scheinen Normkodifizierungen offensichtlich besonders relevant zu sein. Darüber hinaus wurden - oft unter ganz anderen Vorzeichen - Kodifizierungen stilbzw. rhetorikorientiert, also letztlich in pragmatischer Perspektive thematisiert (z.B. Antos 1995; Bremerich-Vos 1991). Relativ selten kommt es vor, dass Autoren, die als Verfasser oder Überarbeiter bei maßgeblichen Kodifizierungen mitgewirkt haben, ausführlich über ihre Arbeit berichten und die Grundsätze ihrer Entscheidungen erläutern (z.B. Eisenberg 2007; ScholzeStubenrecht 1995, 2002).

Ganz allgemein können die im Weiteren zu erläuternden Dimensionen der Sprachkodexforschung wie folgt veranschaulicht werden:

Zum Vergleich ließe sich die anglistische Sprachwissenschaft heranziehen, in der Arbeiten, die explizit den Kodex bzw. präskriptive Diskurse thematisieren, etablierter sind als in der Sprachwissenschaft des Deutschen, vgl. nur zuletzt Schröder/Busse/Schneider (2012), Anderwald $(2011,2012)$. 


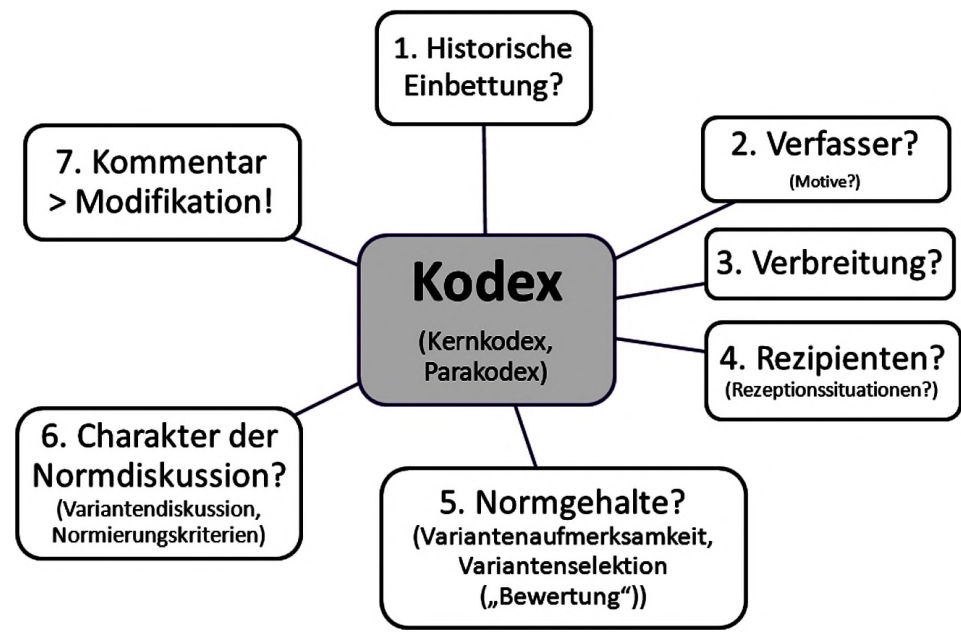

Abb. 1: Dimensionen der Sprachkodexforschung

Bei der Frage nach der historischen Einbettung (1.) geht es darum zu klären, in welchen Beziehungen die Kodex-Texte zu übergreifenden realgeschichtlichen Tatsachen stehen. Das kann - je nach historischem Rahmen - herrschaftsgeschichtlich, politikgeschichtlich, sozialgeschichtlich, kulturgeschichtlich, bildungsgeschichtlich oder mentalitätsgeschichtlich fokussiert werden. Hier ist etwa daran zu denken, dass die Häufung sprachkodifizierender Schriften in der zweiten Hälfte des 19. Jahrhunderts sicher vor dem Hintergrund der Entstehung des deutschen Nationalstaats zu sehen ist. Die Kodifizierung des Deutschen war ein Bestandteil in der Herausbildung eines modernen Staatsbewusstseins. In anderen Konstellationen kann die Kodifizierung z.B. im Rahmen der Erstverschriftlichung einer Sprache oder in der Abwehr von existenzbedrohenden zentrifugalen Kräften eine Rolle spielen.

Was die Verfasser (2.) der Kodex-Texte angeht, so lässt sich z.B. die Frage stellen, welchen Status die Beschäftigung mit Sprache in ihrem Leben spielt. Handelt es sich etwa um Menschen, deren Existenz im weitesten Sinne mit professioneller Sprachbeherrschung verbunden ist, z.B. bei Journalisten, Schriftstellern, Bibliothekaren, Sekretären? Gibt es didaktische Komponenten, z.B. bei einer Anstellung an Schulen, Universitäten oder anderen Bildungsinstitutionen wie Museen oder Bibliotheken? Wie sieht es mit der Nähe oder Distanz der Textautoren zur Sprachwissenschaft der Zeit aus? Oder haben sich vielleicht interessierte, sprachbegeisterte Laien aus mehr oder weniger unerfindlichen Gründen an der Kodifizierung von Sprache beteiligt? Zudem wird es hier wichtig sein, nach den übergeordneten Motiven zu fragen, die die jeweiligen Verfasser zur Abfassung der Kodex-Texte inspiriert haben. Das Motiv-Spektrum ist weit. Im Bewusstsein der Akteure 
kann Dienst an der Sprache, der Sprachgemeinschaft relevant sein, aber auch eine eher abstrakte Orientierung an Verständigungssicherung und Vereinheitlichungslust, bis hin zur sozialsymbolischen Existenzsicherung, buchhändlerischen Geschäftigkeit und purer Besserwisserei. Möglicherweise sind Wustmann und Engel, zwei Autoren einschlägiger Schriften des neuhochdeutschen Parakodex (Meyer 1993; Sauter 2000), gute Beispiele dafür, welche Dinge zusammenfließen müssen, um einer bestimmten Form von Kodifizierung ein relativ großes gesellschaftliches Echo zu verschaffen.

Die Verbreitung (3.) des Kodex möchte ich hier zusammen mit den Rezipienten (4.) und den einschlägigen Rereptionssituationen thematisieren. Für die Einschätzung der Wirkungsmächtigkeit eines Sprachkodex ist es zweckdienlich, danach zu fragen, wie die präskriptiven Kodexinhalte überhaupt verbreitet werden und wer sie in welchen typischen Rezeptionssituationen zur Kenntnis nimmt und womöglich sprachlich realisiert. Es geht also um den möglichen Erfolg von Kodifizierungen. Auch wenn sich hier in historischer Sicht sicherlich nicht alle Fragen klären lassen, so muss man doch davon ausgehen, dass die Forschung an diesem Punkt noch ein weites unbeackertes Feld vor sich hat. Es mag etwa genauer erhoben werden, wie Kodexinhalte im Schulunterricht (z.B. Davies 2000; Dovalil 2011; Schneider 2011) und im öffentlichen Sprachdiskurs verbreitet werden. Oft, aber nicht immer ist damit zu rechnen, dass es primär um Sprachproduktionssituationen geht, in denen die (deutsche) Sprache geschrieben (und dann gelesen) wird. Rezeptionsanalytisch ist es wichtig zu ermitteln, ob es bestimmte gesellschaftliche Gruppen gibt, die die Kodexinhalte stärker als andere Gruppen zur Kenntnis nehmen. Mit einer nachdrücklicheren Rezeption könnten sodann besondere (soziale, kulturelle, individuelle) Vorteile verbunden sein. Der Charakter dieser Vorteile kann mindestens auf zwei Ebenen identifiziert werden: einerseits real, etwa im Sinne von konkreten soziokulturellen Vergünstigungen (z.B. Zugang zu bestimmten Berufen), oder aber imaginiert, also lediglich individuell-antizipatorisch im einzelnen Sprecherbewusstsein, das auf spätere Vorteile hofft, wenn es den Kodifizierungsgehalten folgt.

Bei der Thematisierung der Normgehalte (5.) befinden wir uns im sachlichen Zentrum der Sprachkodexforschung. Denn hier müssen im Detail die jeweiligen sprachlichen Einheiten identifiziert werden, auf die sich die Kodifizierung richtet. Das können - um nur ganz wenige Beispiele zu nennen - konkrete Probleme der Getrennt-Zusammen-Schreibung (Graphematik), der Fremdwortaussprache (Phonetik), der Verbformenbildung (Flexionsmorphologie), der Wortstellung (Syntax), der Nutzung bestimmter Verbbedeutungen (Wortsemantik), des Textaufbaus (Textlinguistik) oder des Gebrauchs von Anredeformen (Pragmatik) sein. Von besonderer Bedeutung ist hier die Frage danach, ob und, wenn ja, auf welche Art und Weise die in jeder Sprache gegebene Variantenvielfalt in den Blick gerät und ggf. mit 
Wertungen zu einzelnen Varianten verbunden wird. Die erste, allem anderen vorgelagerte Frage bezieht sich insofern darauf, ob die Kodifizierer überhaupt Variation wahrnehmen und, wenn ja, welche.

In diesem Zusammenhang möchte ich den Begriff der Variantenaufmerksamkeit prägen. Darunter soll das Ob, das Wie sowie das Ausmaß der Beschäftigung mit sprachlichen Varianten verstanden werden: Welche Varianten geraten überhaupt aus welchem Anlass in den Blick der Kodifizierer? Was wird demgegenüber als stabil und einheitlich gesehen und daher kaum, nicht selten: gar nicht thematisiert? Es ist ja keineswegs so, dass die Bewusstwerdung sprachlicher Varianten einen naturwüchsigen Vorgang darstellt. Vielmehr sollte man in der Thematisierung von Varianten einen metasprachlichen, oft sozial aufgeladenen Konstruktionsprozess sehen, der in verschiedenen Stufen abläuft: Zunächst muss man sprachliche Varianten als solche überhaupt erst einmal wahrnehmen und darüber hinaus als erörterungswürdig ansehen. Dann folgt - auch in der nicht-sprachwissenschaftlichen Sicht - in der Regel die Frage danach, was den Gebrauch der einzelnen Varianten steuert, durch welche Faktoren die Existenz der Varianten also bedingt sein könnte. Aus der Perspektive der Sprachproduktion des Individuums handelt es sich hier also um konditionierte Zweifelsfälle: Die diatopisch, diaphasisch und diastratisch gesteuerten Variationen mögen dabei noch relativ einfach greifbar sein. Für viele Sprecher gehört es vermutlich zur lebensweltlichen Grunderfahrung, dass sich die deutsche Sprache verändert, wenn man auf Reisen unterwegs ist oder in unterschiedlichen Gesprächssituationen oder Gruppenkonstellationen kommuniziert. Schwieriger zu erfassen sind semantische, syntaktische und morphologische Regularitäten bei der Nutzung von Varianten. Ein besonderes Problempotenzial bieten zuletzt diejenigen sprachlichen Varianten, bei denen die Steuerungsfaktoren (noch) gar nicht oder nur dunkel und einigermaßen spekulativ sichtbar werden. In einem solchen Fall stehen die Varianten einfach im Raum, und man kommt bei der Frage, welche Variante man nutzen sollte, nicht so recht weiter: Es handelt sich um echte Zweifelsfälle. ${ }^{2}$

Erst wenn Varianten wahrgenommen und tatsächlich als solche bewusst werden, sie also als Objekte vergegenständlicht wurden, kann im Einzelfall eine Evaluation der Varianten ins Spiel kommen. Der Variantenaufmerksamkeit folgt die bewertende Variantenselektion; sie ist explizit oder implizit angelegt. Für die Analyse der kodifizierten Normgehalte muss also erhoben werden, welche sprachlichen Einheiten mit welchen Wertungsaspekten präsentiert werden. Schon ein flüchtiger Blick in Kodex-Texte zeigt, dass sich derartige Wertungen längst nicht nur in bipolaren Adjektiven wie falsch, unkorrekt, schlecht versus richtig, korrekt, gut verkörpern. Tatsächlich ist davon

Zur Unterscheidung von konditionierten und echten (bzw. unkonditionierten) Zweifelsfällen vgl. Klein (2009, S. 150 ff.). 
auszugehen, dass die Bewertungen in einem Kodex oft auch graduell formuliert werden: Eine Variante ist besser, schlechter, korrekter als eine andere, vielleicht auch noch möglich, nicht gan walsch oder einigermaßen nichtig. Genauso wie der Sprachgebrauch oft durch Skalen und Graduierbarkeit geprägt ist, sind auch Kodex-Texte nicht immer nur dichotom organisiert. Ferner kann das Bewertungsvokabular ganz unterschiedlich schattiert sein. Neben der eher neutralen grammatischen Richtigkeit bzw. Unrichtigkeit können hier verschiedene Wege der Intensivierung bis hin zur emotionalen Aufladung betreten werden. Insbesondere im Parakodex ist etwa der Reigen abwertender Adjektive groß und bunt. Man denke nur an Wörter des folgenden Typs, die man seit dem 19. Jahrhundert in kodifizierenden Texten finden kann. Eine sprachliche Variante ist dann abscheulich, blödsinnig, drollig, einfältig, garstig, geschmacklos, krampfhaft, lächerlich, matt, nachlässig, schleppend, tadelnswert, ungerechtfertigt, unschön, qweifelhaft.

Am Ende dieses Teils der Sprachkodexforschung müsste also jeweils eine lange Liste mit sprachlichen Phänomenen stehen, die im Kodex in irgendeiner Form evaluiert werden: Positiv gesprochen wären die Selektionen zu ermitteln, negativ die Stigmatisierungen (bzw. früher eher „,Diskriminierungen", z.B. Sandig 1973) einzelner sprachlicher Einheiten. Für den Rahmen der jüngeren deutschen Sprachgeschichte ließe sich hier auch unmittelbar an die einschlägige Redeweise von der „Vertikalisierung des Variantenspektrums" (Reichmann 1988; exemplarisch zur Adjektivflexion Voeste 1999) anknüpfen. Anders gesagt: Die Sprachkodexforschung widmet sich hier der Detail-Rekonstruktion des normativen Sprachbewusstseins, insofern es sich in maßgeblichen metasprachlichen Texten manifestiert und zur Auf- bzw. Abwertung bestimmter sprachlicher Einheiten beiträgt. Was etwa für das Hilfsverb tun, den adnominalen Dativ oder das Verhältnis der Diminutionsmorpheme -lein und -chen schon weitgehend geleistet wurde (Langer 2001; Davies/Langer 2006), müsste auch an vielen anderen Stellen noch in allen Einzelheiten herausgeschält werden.

Dabei sei auch auf den Umstand hingewiesen, dass der Sprachkodex in einer bestimmten Zeit durchaus nicht immer mit einer Zunge sprechen muss. Es ist zwar gut möglich, dass Kern- und Parakodex (bzw. die KodexTexte insgesamt) oft in eine Richtung weisen. Daneben ist aber auch damit zu rechnen, dass die Bewertungen des einen Kodex-Texts nicht mit der Bewertung eines anderen übereinstimmen. Wie es im Sprachusus selber Schwankungen gibt, so können auch im Sprachkodex Schwankungen existieren. Auf die maßgebliche Funktionalität des Kodex für die Herausbildung von Standardvarietäten bezogen: In der Regel herrscht sowohl im Usus als auch im Kodex einer Standardsprache ein gewisses Maß an Uneinheitlichkeit. Das sollte bei der oft vorschnell angesetzten „Einheitlichkeit“ der Standardsprache nicht übersehen werden. Von daher ist es auch eine besonders interessante Aufgabe der Sprachkodexforschung herauszufinden, in- 
wiefern und an welchen Stellen der Sprachkodex zu einer bestimmten Zeit eher von Homogenität oder von Inhomogenität geprägt ist.

Die Beschäftigung mit den konkreten Normgehalten involviert die systematische Erörterung der jeweiligen Norm-bzw. Variantendiskussion (6.). Es geht also darum, den diskursiven Charakter der metasprachlichen Thematisierung sprachlicher Variation zu erforschen. Dabei kann sich der Blick auf jede einzelne sprachliche Einheit richten, insbesondere darauf, durch welche Bewertungsaspekte und Diskussionsinhalte die Varianten normativ unterschieden und mit bestimmten Gehalten aufgeladen werden. Auch hier ist das Spektrum der möglichen Befunde groß: Einerseits ist oft damit zu rechnen, dass überhaupt keine explizite Diskussion der Normierungskriterien erfolgt und ein bestimmtes sprachliches Phänomen schlicht als gut (o.ä.) oder schlecht (o.ä.) herausgestellt wird. Andererseits können in einem Kodex-Text auch sehr unterschiedliche Begründungen für die Favorisierung oder Verunglimpfung einzelner sprachlicher Varianten gegeben werden, etwa sprachsystembezogene (z.B. durch Herausstellung von Analogien) (Lühr 1992), kommunikationsorientierte (z.B. durch Thematisierung von „Verständlichkeit" oder pragmatischer Angemessenheit), sprachhistorische (z.B. durch Bezüge auf angeblich ,ursprüngliche "Sprachzustände) oder sprachlandschaftlich angelegte, personen- bzw. autoritätsbezogene (z.B. ,gute Schriftsteller") oder ästhetische Argumente. In jedem Fall wäre an diesem Punkt anzustreben, die inhaltlichen und formalen Muster der metasprachlichen Normbegründung innerhalb eines Kodex präzise und detailliert zu rekonstruieren und zu interpretieren. Insbesondere die Kodifizierungen des 17. und 18. Jahrhunderts wurden in dieser Dimension schon vergleichsweise genau aufgearbeitet (z.B. Josten 1976; FaulstichChrist 2008; Lange 2008). Diese frühmoderne Geschichte ist auch deshalb von Bedeutung, weil die Normdiskussionen und Normgehalte des 19. und 20. Jahrhunderts oft auf älteren Vorgaben beruhen. Allerdings tauchen dann im Zuge der historischen Entwicklung Unterschiede in den Normierungsdiskursen auf, die es vorher in dieser Form nicht gegeben hat (z.B. Law 2006).

Vor diesem Hintergrund ließe sich nicht zuletzt danach fragen, wie sich die Kodifizierungsinhalte und -aktivitäten zu den übergreifenden Entwicklungsprozessen einer Sprache verhalten. Zunächst scheint plausibel, dass Kodifizierungen durch ihre bloße Anlage einen konstitutiv konservativen Charakter besitzen. Kodifizierungen präferieren bei der Thematisierung von Varianten gegebenenfalls eher die alte als die neue Variante. Aber ist das immer und unterschiedslos auf allen sprachlichen Ebenen so? Und welche Faktoren können dazu führen, dass auch die neuen Varianten im Kodex akzeptiert werden? Kodizes sind ja ihrerseits nicht völlig stabil, sondern reagieren - wenn auch verzögert - auf Sprachwandelprozesse. Unter welchen Bedingungen? In welchen Formen? Mit welchen Konsequenzen? 
Entsprechende Untersuchungsperspektiven lassen sich auch auf die aktuellen Forschungen zu übergreifenden Sprachwandelvorgängen beziehen. Besonders interessant wäre es sicherlich, systematisch der Frage nachzugehen, in welchen Formen und Mustern der Umbau von einer Silbensprache zu einer Wortsprache in den Kodifizierungen begleitet wird. ${ }^{3}$ Wirken die Kodifizierungen eher entwicklungsunterstützend oder entwicklungshemmend? Wann und unter welchen Bedingungen werden die sich allmählich durchsetzenden Varianten akzeptiert? Dasselbe ließe sich mit Blick auf den älteren Topos des Übergangs von einer synthetisch zu einer analytisch angelegten Sprache fragen. An welchen Punkten präferieren die Kodifizierungen eher alte, synthetische Baumuster, wo neue, analytische Sprachformen? Auch Einzelaspekte der Sprachentwicklung sind für solche Fragerichtungen zugänglich: Welche Positionen beziehen Kodifizierungen etwa gegenüber der Integration von Fremdwörtern in die deutsche Sprache? Wo, wann, mit welcher Nachdrücklichkeit und unter welchen Umständen agieren sie gegen bzw. für die Integration „,fremder" Einheiten ins Deutsche? Derartige Fragen laufen am Ende in einem übergeordneten Problemkomplex zusammen, der - sicher sehr verkürzt - auf die folgende Formel zu bringen ist: Hätte die deutsche Sprache der Gegenwart eine andere Gestalt, wenn es in der Vergangenheit keine oder eine andere Kodifizierung gegeben hätte? Wenn man vorab schon von einer gewissen Wirkungskraft der Kodizes überzeugt ist, ließe sich die Frage auch anders stellen: Welchen Anteil an der historischen Prägung der deutschen Sprache besaßen die diversen Kodifizierungen? Genauer: An welchen Stellen besitzen sie günstige Auswirkungen auf sprachliche Phänomene, die in einem nicht-kodifizierenden Umfeld vermutlich ohne Durchsetzungskraft geblieben wären?

Der letzte Punkt der Sprachkodexforschung, also Kommentar und ggf. Modifikation (7.), bezieht sich weniger auf den Sprachkodex an und für sich, sondern auf seine öffentliche Relevanz. Denn je nachdem, wie und mit welcher Stoßrichtung in der gesellschaftlichen Diskussion Kodex-Probleme thematisiert werden, ist es nötig, sprachwissenschaftlich Stellung zu beziehen. In einem Sprachkodex können nämlich falsche, schiefe, unklare oder unpassende Dinge kodifiziert sein. Gegebenenfalls sind also im nicht-sprachwissenschaftlichen Raum korrigierende Kommentare zu Kodifizierungen nötig, manchmal selbst Plädoyers für Modifikationen. Auch damit eröffnet sich wieder ein weites Feld, dazu nur zwei Illustrationen: In der jüngsten Zeit kann man etwa auf die verschiedenen Beiträge und Kommentare zu den Para-Kodifizierungen von Bastian Sick hinweisen (Maitz/Elspaß 2007; Meinunger 2008; Hundt 2010). Während es sich bei diesen Stellungnahmen um explizite Kodex-Kommentare handelt, können andere Einwürfe auch einen impliziteren Zuschnitt besitzen. Wer beispielsweise angesichts der Ko-

Zu dieser Forschungsperspektive vgl. die Beiträge von Nübling, Dammel und Szczepaniak (in diesem Band). 
difizierungsproblematik auf dem seit jeher brisanten Feld der Orthographie die Redeweise vom „Charme der Freiheit" (Eichinger 2011, S. 50) ins Spiel bringt, nimmt mittelbar dazu Stellung, wie der Kodex der gegenwärtigen Standardsprache an bestimmten Stellen beschaffen sein sollte. Dieser letzte Punkt der Sprachkodexforschung lässt sich am Ende auf eine einfache Formel bringen: Die Sprachwissenschaft nimmt hier ihre gesellschaftliche Verantwortung wahr und beteiligt sich - zumindest kommentierend - an dem Projekt der angemessenen und wissenschaftlich haltbaren Kodifizierung einer Sprache.

\section{Einige Schlaglichter zum neuhochdeutschen Grammatik-Kodex seit dem 19. Jahrhundert}

In den obigen Kapiteln wurde eher theoretisch und programmatisch zum Problem des Sprachkodex Stellung genommen. Zum Abschluss möchte ich dagegen einige Bemerkungen dem neuhochdeutschen Sprachkodex im besonderen widmen. Ausgehend von heute lässt sich zunächst eine zentrale Schrift des gegenwärtigen Kern-Kodex etwas näher beleuchten. Ich meine die Duden-Grammatik, die die Fortsetzung der Grammatik von Friedrich Bauer (1812-1874) darstellt. Letztere erschien bereits im 19. Jahrhundert in vielen Auflagen. Sie sprechen für eine große Verbreitung des Buchs. Exemplarisch können drei unterschiedliche Auflagen herangezogen werden, nämlich die aus den Jahren 1850, 1871 (13. Aufl.) und 1912 (27. Aufl., Bearb. Konrad Duden). Wie sah in diesen Kodex-Texten die Variantenaufmerksamkeit aus? Welche Varianten gerieten in den Blick des Kodifizierers? Die Antworten auf diese Frage stehen in der Tabelle 1. Sie verzeichnet (mit Kreuz), welche Variantenpaare im jeweiligen Text aufgegriffen wurden und welche nicht (kein Kreuz):

\begin{tabular}{|l|l|c|c|c|}
\hline \multicolumn{5}{|c|}{ Bauer-Duden-Tradition } \\
\hline $\begin{array}{l}\text { Grammatisches } \\
\text { Phänomen }\end{array}$ & \multicolumn{1}{|c|}{ Beispiel } & 1850 & 1870 & 1912 \\
\hline $\begin{array}{l}\text { Substantivflexion: } \\
\text { Genitiv-s }\end{array}$ & Tages/Tags & & $\begin{array}{c}\mathbf{x} \\
(\$ 29)\end{array}$ & $\begin{array}{c}\mathbf{x} \\
(\mathrm{S} .28)\end{array}$ \\
\hline Substantiv: s-Plural & Jungen/Jungens & & $\begin{array}{c}\mathbf{x} \\
(\$ 28)\end{array}$ & $\begin{array}{c}\mathbf{x} \\
(\mathrm{S} .28)\end{array}$ \\
\hline Substantiv: Plural & Gastmable/Gastmähler & & $\begin{array}{c}\mathbf{x} \\
(\$ 31)\end{array}$ & $\begin{array}{c}\mathbf{x} \\
(\mathrm{S} .27)\end{array}$ \\
\hline NP: Maßangaben & $\begin{array}{l}\text { zwei Flaschen süßer/-n } \\
\text { Wein/-s }\end{array}$ & & & $\begin{array}{c}\mathbf{x} \\
(\mathrm{S} .37 \mathrm{f})\end{array}$ \\
\hline
\end{tabular}




\begin{tabular}{|c|c|c|c|c|}
\hline \multicolumn{5}{|c|}{ Bauer-Duden-Tradition } \\
\hline $\begin{array}{l}\text { Grammatisches } \\
\text { Phänomen }\end{array}$ & Beispiel & 1850 & 1870 & 1912 \\
\hline Eigennamen: Genitiv & Klaus'/ Klausens & & $\begin{array}{c}\mathbf{x} \\
(\mathrm{S} .34)\end{array}$ & $\begin{array}{c}\mathbf{x} \\
(\mathrm{S} .35)\end{array}$ \\
\hline Verb: 2. Pers. Sg./P1. & traget/ tragt & $\stackrel{\mathbf{x}}{\left(\int^{5} 57\right)}$ & $\begin{array}{c}\mathbf{x} \\
(\mathbb{S} 58) \\
\end{array}$ & $\begin{array}{c}\mathbf{x} \\
(\mathrm{S} .61) \\
\end{array}$ \\
\hline Verb: Partizip II & $\begin{array}{l}\text { singen bören/singen } \\
\text { gehört }\end{array}$ & & $\begin{array}{c}\mathbf{x} \\
\left(\int 66\right)\end{array}$ & $\begin{array}{c}\mathbf{x} \\
(\mathrm{S} .70)\end{array}$ \\
\hline Verb: Konjunktiv II & stände/ stünde & & $\begin{array}{c}\mathbf{x} \\
(\mathbb{S} 62)\end{array}$ & $\begin{array}{c}\mathbf{x} \\
(\mathrm{S} .66)\end{array}$ \\
\hline $\begin{array}{l}\text { Verb: Perfekt: sein } \\
\text { oder baben }\end{array}$ & $\begin{array}{l}\text { ich bin gesessen } / i c h \\
\text { babe gesessen }\end{array}$ & $\begin{array}{c}\mathbf{x} \\
(\mathbb{5} 56)\end{array}$ & $\begin{array}{c}\mathbf{x} \\
(\mathbb{5} 56)\end{array}$ & $\begin{array}{c}\mathbf{x} \\
(\mathrm{S} .58)\end{array}$ \\
\hline $\begin{array}{l}\text { Adjektiv auf -el/-er/ } \\
\text {-en: Flexionsformen }\end{array}$ & edlen/edeln & & $\begin{array}{c}\mathbf{x} \\
(\mathbb{4} 40)\end{array}$ & $\begin{array}{c}\mathbf{x} \\
(\mathrm{S} .39)\end{array}$ \\
\hline $\begin{array}{l}\text { Adjektiv in NP: } \\
\text { Genitiv }\end{array}$ & $\begin{array}{l}\text { gutes Mutes/guten } \\
\text { Mutes }\end{array}$ & $\begin{array}{c}\mathbf{x} \\
((338)\end{array}$ & $\begin{array}{c}\mathbf{x} \\
(\mathbb{3} 38)\end{array}$ & $\begin{array}{c}\mathbf{x} \\
(\mathrm{S} .40,140) \\
\end{array}$ \\
\hline $\begin{array}{l}\text { Adjektiv in NP } \\
\text { Flexion: Spezialfälle }\end{array}$ & $\begin{array}{l}\text { Liebe Freunde/ lieben } \\
\text { Freunde (Anrede) }\end{array}$ & $\begin{array}{c}\mathbf{x} \\
(\mathbb{1} 112) \\
\end{array}$ & $\begin{array}{c}\mathbf{x} \\
(\mathbb{1} 112) \\
\end{array}$ & $\begin{array}{c}\mathbf{x} \\
(\mathrm{S} .141) \\
\end{array}$ \\
\hline Adjektiv: gereiht & $\begin{array}{l}\text { nach langem schwerem } \\
\text { Leiden/ nach langem } \\
\text { schweren I eiden }\end{array}$ & $\left(\begin{array}{l}\mathbf{x} \\
37\end{array}\right)$ & $\begin{array}{c}\mathbf{x} \\
(0112)\end{array}$ & $\begin{array}{c}\quad \mathbf{x} \\
(\mathrm{S} .142)\end{array}$ \\
\hline $\begin{array}{l}\text { Präpositionen: } \\
\text { Rektion }\end{array}$ & trotz des/trotz dem & $\begin{array}{c}\mathbf{x} \\
(979)\end{array}$ & $\begin{array}{c}\mathbf{x} \\
\left(S^{79}\right)\end{array}$ & $\begin{array}{c}\mathbf{x} \\
(\mathrm{S} .83)\end{array}$ \\
\hline $\begin{array}{l}\text { Pronomen auf -er: } \\
\text { Flexionsformen }\end{array}$ & $\begin{array}{l}\text { unserem/unsrem/ } \\
\text { unserm }\end{array}$ & & $\begin{array}{c}\mathbf{x} \\
(\mathbb{4} 40)\end{array}$ & $\begin{array}{c}\mathbf{x} \\
(\mathrm{S} .44)\end{array}$ \\
\hline
\end{tabular}

Tab. 1: Grammatische Varianten in der Bauer-Duden-Tradition

Die obige Übersicht lässt sich fürs Erste wie folgt interpretieren: Zunächst ist auffällig, dass bereits im 19. Jahrhundert phänomenologisch genau diejenigen Varianten auftauchen, die noch in der grammatischen Variantenaufmerksamkeit der Gegenwart auftauchen. Als Beleg möchte ich diesbezüglich nur auf die einschlägigen Artikel des gegenwärtigen Kern-Kodex verweisen, vor allem auf den so genannten Zweifelsfälle-Duden und das entsprechende Gegenstück aus dem Wahrig-Verlag (Duden 2011; Wahrig 2009). Schon im 19. Jahrhundert finden wir diejenigen Zweifelsfälle, die auch heute noch, z.T. allerdings mit anderen lexikalischen Belegungen, das grammatische Problemfeld dominieren. Die Schlussfolgerungen, die sich daraus ziehen lassen, sind wohl zweideutig. Denn zum einen könnte man 
daraus ableiten, dass die neuhochdeutsche Variantenaufmerksamkeit recht stabil ist. Die neuhochdeutsche Sprache drängt die Sprecher sozusagen an denselben Systemstellen zur metasprachlichen Aufmerksamkeit, weil sie typologisch weitgehend von denselben Brüchen und Klippen beherrscht ist. Die Folge ist: Das normative Problempotenzial hat sich in den letzten 150 Jahren kaum geändert. Andererseits sprechen diese Befunde aber vielleicht nur dafür, dass die gegenwärtigen Kodifizierungen lediglich Fortschreibungen der älteren Texte darstellen. Etwas anders gewendet: Was einmal in das metasprachliche Bewusstsein der Kodifizierer gerät und damit auch das Sprecherbewusstsein formt, tendiert zur Versteinerung. Fine Kodifizierung zieht, so sieht es aus, viele andere, die dann ähnlich gelagert sind, nach sich. Insgesamt verbreitert und verstetigt sich eine einmal in Gang gesetzte Kodifizierungsaktivität. Diese Auslegung wird auch dadurch gestützt, dass die Kodex-Tradition offensichtlich mit einer geringen Variantenaufmerksamkeit einsetzt - viele Variantenpaare tauchen in der 1850er Auflage gar nicht auf -, dann aber 1870 und 1912 recht schnell größere Ausmaße annimmt. Angesichts des anscheinend konstitutiv konservativen Gepräges der Kodifizierung erhebt sich umso mehr die Frage, an welchen Stellen und unter welchen Bedingungen es zu nachhaltigen Änderungen der Normgehalte im Kodex kommt. Im weiteren wäre darüber hinaus zu erheben, ob und, wenn ja, in welchen Formen es bei diesen Varianten zu Selektions- und Evaluationsprozessen gekommen ist.

Dieses noch recht bruchstückhafte und impressionistische Bild der Bauer-Duden-Tradition lässt sich mit vorläufigen Befunden aus einer Untersuchung zur Kodifizierung der deutschen Sprache in Schulgrammatiken des 19. und 20. Jahrhunderts kontrastieren. ${ }^{4}$ Nach der systematischen, statistisch gestützten Detail-Analyse von 40 Schulgrammatiken zeichnet sich folgendes Bild ab: Zunächst ist festzustellen, dass sprachliche Varianz in den Schulgrammatiken seit Beginn des 19. Jahrhunderts in der einen oder anderen Form thematisiert wird. Es ist also nicht so, dass die Autoren der Schulgrammatiken der sprachlichen Varianz aus dem Wege gegangen wären. Sie waren keine varietätenlinguistischen Ignoranten. Fragt man nach möglichen Verschiebungen im Laufe des Jahrhunderts, so ergibt sich der Befund, dass der Umfang der aufgegriffenen Varianz zum Ende des Jahrhunderts deutlich abnimmt - ein Ergebnis, das eher im Widerspruch zu dem oben angesprochenen Verlaufsmuster in der Bauer-Duden-Tradition steht, wo die Thematisierung der Varianz eher zunimmt. Seit den 1890er Jahren wird in den Schulgrammatiken gut ein Drittel weniger Varianz aufgenommen als

Im Folgenden beziehe ich mich auf die ersten, vorläufigen Resultate einer Dissertation, die Dominik Banhold derzeit an der Universität Würzburg unter dem Arbeitstitel „Sprachnorm, Sprachbewertung, Sprachlehre. Zum Ungang mit flexionsmorphologischer Varianz in deutschen Schulgrammatiken (1800-1939)" erhoben hat. Ich danke dem Autor für die Erlaubnis, diese Ergebnisse hier zu referieren. 
zuvor. Dem korrespondiert freilich eine nicht zu übersehende deskriptive Komponente. Denn insgesamt werden in knapp 50\% der Fälle die entsprechenden Varianten als gleichwertig dargestellt, ohne dass in irgendeiner Form Markierungen, seien es Bewertungen oder Angaben zum Gebrauchskontext, vorlägen. Anders gesagt: bei vielen Variantenthematisierungen greift in den Schulgrammatiken keine (1) Variantenselektion, da die Existenz der Varianten nur konstatiert und keine der Varianten normativ präferiert wird. Nicht immer ist also eine der Varianten ,fehlerhaft ${ }^{c c}$. In der zweiten Hälfte und besonders zum Ende des 19. Jahrhunderts nehmen darüber hinaus Angaben zum Gebrauchskontext der Varianten deutlich zu. Man könnte also von der Zunahme pragmatischer Relativierungen und Konditionierungen sprechen. Gleichzeitig verringert sich die Anzahl normativer Stellungnahmen in Form von vertikalisierenden Bewertungen. Insgesamt überwiegen freilich die neutralen Zugriffe und Angaben zum Gebrauchskontext vor vertikalisierend-selektiven Bewertungen.

Diese Befunde und Interpretationen zum neuhochdeutschen Sprachkodex müssen selbstverständlich weiter und genauer ergänzt werden. Dazu sind andere Untersuchungen zum neuhochdeutschen Kern- und Parakodex vonnöten, in denen sämtliche Systemebenen und Gebrauchsformen des Deutschen in allen relevanten Details und in größeren Zusammenhängen zu beleuchten sind. In diesem Kontext sei am Ende auf eine elektronische Unterstützung für entsprechende Forschungen hingewiesen. Im Würzburger ZweiDat-Projekt werden zentrale Texte des neuhochdeutschen Sprachkodex systematisch aufgearbeitet und online verfügbar gemacht (www.zweidat. germanistik.uni-wuerzburg.de). Mit der Hilfe dieser Datenbank können die Kodex-Texte schnell und perspektivreich nach verschiedenen Kriterien durchsucht werden. Dazu stehen etwa Suchroutinen zur Verfügung, die sich auf die einschlägigen grammatischen Phänomene und Beispielwörter beziehen. So lassen sich z.B. schnell diejenigen Kodex-Passagen einsehen, die dem Pro und Kontra des Dativ-e gewidmet sind. Darüber hinaus ist auch eine Recherche zu den jeweiligen Normdiskussionen möglich. So lässt sich etwa rasch ermitteln, in welchen Kodex-Abschnitten auf den Sprachgebrauch von Adelung, Schiller oder Fontane referiert wurde. Dadurch werden Detailerhebungen zum neuhochdeutschen Kodex möglich. Auf Goethe etwa bezog man sich nicht nur als sprachliches Vorbild, sondern auch als abschreckendes Beispiel. ${ }^{5}$ Die klassischen Schriftsteller verkörperten also keine sakrosankten Ideale.

5 Vg-1. z.B. bei der Diskussion der relativischen Anschlüsse das vs. was: „Daß aber was auch noch darüber hinaus auch auf sächliche Hauptwörter bezogen wird, kann als gänzlich seiner Bedeutung widersprechend dadurch nicht schriftgemäß werden, daß man dies gedankenlos immer häufiger mitmacht, wie es denn auch Goethe und später G. Freytag hierin wiederholt versehen haben [...]. Also sage niemand mit Goethe: das Bücblein, was, sondern das [...]." (Matthias 1892, \$100). 
Vergleichbares gilt für den Bezug auf diatopische Instanzen (Sprachgebrauch in Berlin, Frankfurt, Norddeutschland, Österreich usw.), diastratische, mediale und funktionale Bezugspunkte (Sprachgebrauch der Gebildeten, der Ungelehrten, des Volks, der Schrifttexte, der Zeitungen, der Dichter, der Kanzleien, der Geschäftsleute usw.) und diachrone Befunde (Sprache im Mittelalter, im 16., 17. oder 18. Jahrhundert usw.).

\section{Fazit}

In diesem Text habe ich dafür plädiert, die Erforschung von Sprachkodifizierungen stärker als bisher ins Zentrum der Sprachwissenschaft zu rücken. Diesem Ziel diente zunächst eine Definition des Terminus Sprachkodex: Seine Reichweite bezieht sich einerseits auf Texte, die auch in der Vergangenheit schon in den Blick genommen wurden (= Kernkodex). Darüber hinaus wurden hier aber auch andere, bisher kaum systematisch erfasste Texte als kodifizierende Instanzen gesehen (= Parakodex). Auf dieser Basis ergaben sich einige Perspektiven der Sprachkodexforschung, die mit Beispielen aus der Erforschung des neuhochdeutschen Sprachkodex illustriert wurden. Insgesamt sollte deutlich geworden sein, dass die Ideen zur Sprachkodexforschung selbstverständlich nicht völlig neu sind, sondern ältere Forschungen aufgreifen und weiterführen. Die innovative Komponente dürfte vor allem darin liegen, Sprachkodifizierungen nicht am Rande anders akzentuierter Untersuchungen flankierend mit zu behandeln, sondern in den Mittelpunkt zu stellen und systematisch mit übergreifenden sprachwissenschaftlichen Fragestellungen zu verbinden.

Im Lichte dieser Ausführungen lässt sich dann auch die Frage aus der Überschrift dieses Texts aufnehmen: Gibt es einen Kodex für die Grammatik des Neuhochdeutschen? Ja, natürlich gibt es einen Kodex der neuhochdeutschen Sprache. Er zerfällt aber mindestens in zwei große Teile, nämlich den Kern- und den Parakodex. In beiden Fällen handelt es sich jeweils um ein Konglomerat von kodifizierenden Texten. Der Sprachkodex ist letztlich unübersichtlicher und vielgestaltiger, als es bisher oft angenommen wurde, wenn mit einem quasi-juristischen Kodex-Begriff gearbeitet wurde. Die verschiedenen Kodex-Texte weisen vermutlich oft in dieselbe Richtung, können aber auch von Inhomogenitäten und Wandelprozessen geprägt sein. Diese Verhältnisse zu entwirren, die Resonanz des Kodex in der Sprach- und Gesellschaftsentwicklung genau zu eruieren und am Ende für einen sachhaltigen, angemessenen Sprachkodex zu sorgen, ist Aufgabe der Sprachkodexforschung.

Für die Suggestionen, die im öffentlichen Diskurs über den angeblichen deutschen Sprachverfall verkündet werden, könnte aus der forcierten Forschung am Ende eine ebenso substanzielle wie facettenreiche Antwort auf 
eine einfache Frage resultieren: Ist es wirklich so, dass wir heute den (kodifizierten!) Sprachregeln nicht mehr folgen und die deutsche Sprache daher auf dem absteigenden Ast ist? Ohne den zukünftigen Untersuchungen zu sehr vorgreifen zu wollen, möchte ich auf diese Frage eine hypothetische Antwort wagen: Nein, so ist es nicht; in den allermeisten Fällen folgen wir weiterhin den Sprachregeln. Und wenn die deutsche Sprachgemeinschaft doch einmal auf breiter Front eine Regel brechen sollte, so ist die Kodifizierung zu modifizieren - und der Einklang zwischen Sprachgebrauch und Sprachregel ist wiederhergestellt. Man folgt eben neuen Regeln. Kurzum: Ein Sprachverfall durch Regelbruch findet nicht statt.

\section{Literatur}

Ammon, Ulrich (1986): Explikation der Begriffe Standardvarietät und Standardsprache auf normtheoretischer Grundlage. In: Holtus, Günter/Radtke, Edgar (Hg.): Sprachlicher Substandard. (= Konzepte der Sprach- und Literaturwissenschaft 36). Tübingen, S. 1-64.

Ammon, Ulrich (1995): Die deutsche Sprache in Deutschland, Österreich und der Schweiz. Das Problem der nationalen Varietäten. Berlin.

Ammon, Ulrich (2005): Standard und Variation: Norm, Autorität, Legitimation. In: Eichinger, Ludwig M./Kallmeyer, Werner ( $\mathrm{Hg}$.): Standardvariation. Wie viel Variation verträgt die deutsche Sprache? (= Jahrbuch des Instituts für Deutsche Sprache 2004). Berlin/New York, S. 28-40.

Anderwald, Lieselotte (2011): Norm vs variation in British English irregular verbs: the case of past tense sang vs sung. In: English Language and Linguistics 15, S. 85-112.

Anderwald, Lieselotte (2012): Clumsy, awkward or having a peculiar propriety? Prescriptive judgements and language change in the 19th century. In: Language Sciences 34, S. $28-53$.

Antos, Gerd (1995): Warum gibt es normative Stilistiken? Sprachtheoretische Überlegungen zu einem scheinbar trivialen Problem. In: Stickel, Gerhard ( $\mathrm{Hg}$ ): Stilfragen. (= Jahrbuch des Instituts für deutsche Sprache 1994). Berlin/New York, S. 355-377.

Bauer, Friedrich (1850): Grundzüge der Neuhochdeutschen Grammatik für die unteren und mittleren Klassen höherer Bildungsanstalten. Nördlingen.

Bauer, Friedrich (1871): Grundzüge der Neuhochdeutschen Grammatik für höhere Bildungsanstalten. 13. Aufl. München.

Bauer, Friedrich (1912): Grundzüge der Neuhochdeutschen Grammatik für höhere Bildungsanstalten und zur Selbstbelehrung für Gebildete. Bearbeitet von Konrad Duden. 27. Aufl. München.

Beck, Götz (1996): Sprachkritik - „Sprachverfall“. Zur Phänomenologie einer Sprachverwirrung. In: Peyer, Ann/Portmann, Paul R. (Hg): Norm, Moral und Didaktik - Die Linguistik und ihre Schmuddelkinder. Eine Aufforderung zur Diskussion. Tübingen, S. 61-95. 
Bittner, Andreas/Köpcke, Klaus-Michael (2008): Sprachwandel- oder Verlotterungsprozesse. Versuch einer Versachlichung. In: Denkler, Markus (Hg:): Frischwärts und unkaputtbar. Sprachverfall oder Sprachwandel im Deutschen. Münster, S. 59-80.

Bremerich-Vos, Albert (1991): Populäre rhetorische Ratgeber. Historisch-systematische Untersuchungen. (= Reihe germanistische Linguistik 112). Tübingen.

Davies, Winifred V. (2000): Linguistic norms at school: A survey of secondary-school teachers in a Central German dialect area. In: Zeitschrift für Dialektologie und Linguistik 67, S. 129-147.

Davies, Winifred V./Langer, Nils (2006): The making of bad language. Lay Linguistic Stigmatisations in German: Past und present. (= VarioLingua 28). Frankfurt a.M.

Dovalil, Vit (2006): Sprachnormenwandel im geschriebenen Deutsch an der Schwelle zum 21. Jahrhundert. Die Entwicklung in ausgesuchten Bereichen der Grammatik. (= Duisburger Arbeiten zur Sprach- und Kulturwissenschaft 63). Frankfurt a.M.

Dovalil, Vit (2011): Sprachnormen im Schulunterricht: Eine Untersuchung aus soziolinguistischer Perspektive. In: Lejsková, Alena/Valdrová, Jana (Hg.): Die Grammatik, Semantik und Pragmatik des Wortes. Ihre Erforschung und Vermittlung. (= Budweiser Arbeiten zur Germanistik in Unterricht und Forschung 1). Augsburg, S. 66-88.

Duden (2011): Der Duden: Bd. 9: Richtiges und gutes Deutsch. Das Wörterbuch der sprachlichen Zweifelsfälle. Hrsg. u. überarb. v. d. Dudenredaktion. Unter Mitw. v. Peter Eisenberg und Jan Georg Schneider 7., vollst. überarb. Aufl. Mannheim u.a.

Ehrlich, Karoline (2008): Wie spricht man „richtig ${ }^{c}$ Deutsch? Kritische Betrachtung der Aussprachenormen von Siebs, GWDA und Aussprache-Duden. Wien.

Eichinger, Ludwig M. (2011): Normprobleme, oder: Variation ist sinnvoll. Überlegungen zum heutigen Deutsch. (= Abhandlungen der Geistes- und Sozialwissenschaftlichen Klasse 2011, 6). Stuttgart.

Eisenberg, Peter (2007): Sprachliches Wissen im „Wörterbuch der Zweifelsfälle“. Über die Rekonstruktion einer Gebrauchsnorm. In: Aptum. Zeitschrift für Sprachkritik und Sprachkultur 3, S. 209-228.

Elspaß, Stephan (2005): Language norm and language reality. Effectiveness and limits of prescriptivism in New High German. In: Langer, Nils/Davies, Winifred V. (Hg.): Linguistic purism in the Germanic Language. (= Studia Linguistica Germanica 75). Berlin/New York, S. 20-46.

Faulstich-Christ, Katja (2008): Konzepte des Hochdeutschen. Der Sprachnormierungsdiskurs im 18. Jahrhundert. (= Studia Linguistica Germanica 91). Berlin.

Görtz, Barbara (1990): Untersuchung zur Diskussion über das Thema Sprachverfall im Fin-de-siècle. (= Bonner romanistische Arbeiten 36). Frankfurt a.M. u.a.

Hennig, Mathilde ( $\mathrm{Hg}$ ) (2009): Wie normal ist die Norm? Sprachliche Normen im Spannungsfeld von Sprachwissenschaft, Sprachöffentlichkeit und Sprachdidaktik. Kassel.

Höchli, Stefan (1981): Zur Geschichte der Interpunktion im Deutschen. Eine kritische Darstellung der Lehrschriften von der zweiten Hälfte des 15. Jahrhunderts bis zum Ende des 18. Jahrhunderts. (= Studia Linguistica Germanica 17). Berlin/New York. 
Hollmach, Uwe (2007): Untersuchungen zur Kodifizierung der Standardaussprache in Deutschland. (= Hallesche Schriften zur Sprechwissenschaft und Phonetik 21). Frankfurt a.M. [Zugl.: Habil.-Schr., Univ. Halle-Wittenberg, 2004.]

Hundt, Markus (2000): „Spracharbeit“ im 17. Jahrhundert. Studien zu Georg Philipp Harsdörffer, Justus Georg Schottelius und Christian Gueintz. (= Studia Linguistica Germanica 57). Berlin/New York.

Hundt, Markus (2010): Bastian Sick: Der Dativ ist dem Genitiv sein Tod. In: Mitteilungen des Deutschen Germanistenverbandes 57, 2, S. 174-196.

Josten, Dirk (1976): Sprachvorbild und Sprachnorm im Urteil des 16. und 17. Jahrhunderts. Sprachlandschaftliche Prioritäten Sprachautoritäten, sprachimmanente Argumentation. (= Europäische Hochschulschriften: Reihe 1, Deutsche Literatur und Sprache 152). Bern.

Keller, Rudi (2006): Ist die deutsche Sprache vom Verfall bedroht? In: Aptum. Zeitschrift für Sprachkritik und Sprachkultur 3, S. 193-205.

Klein, Wolf Peter (2003): Sprachliche Zweifelsfälle als linguistischer Gegenstand. Zur Einführung in ein vergessenes Thema der Sprachwissenschaft. In: Linguistik online 16. Internet: www.linguistik-online.org/16_03/klein.html (Stand 8.8.2013).

Klein, Wolf Peter (2004): Deskriptive statt präskriptiver Sprachwissenschaft? Über ein sprachtheoretisches Bekenntnis und seine analytische Präzisierung. In: Zeitschrift für germanistische Linguistik 32, S. 376-405.

Klein, Wolf Peter (2006): Vergebens oder vergeblich? Ein Analysemodell zur Untersuchung sprachlicher Zweifelsfälle. In: Breindl, Eva/Gunkel, Lutz/Strecker, Bruno (Hg.): Grammatische Untersuchungen. Analysen und Reflexionen. Gisela Zifonun zum 60. Geburtstag. (= Studien zur deutschen Sprache 36). Tübingen, S. 581599.

Klein, Wolf Peter (2009): Auf der Kippe? Zweifelsfälle als Herausforderung(en) für Sprachwissenschaft und Sprachnormierung. In: Konopka, Marek/Strecker, Bruno (Hg.): Deutsche Grammatik - Regeln, Normen, Sprachgebrauch. (= Jahrbuch des Instituts für Deutsche Sprache 2008). Berlin/New York, S. 141-165.

Klein, Wolf Peter (2011): Sprachliche Zweifelsfälle im Frühneuhochdeutschen. Alte und neue Perspektiven ihrer Erforschung. In: Lobenstein-Reichmann, Anja/Reichmann, Oskar (Hg.): Frühneuhochdeutsch - Aufgaben und Probleme seiner linguistischen Beschreibung. (= Germanistische Linguistik 213/215). Hildesheim u.a., S. $275-316$.

Klein, Wolf Peter (2013): Warum brauchen wir einen klaren Begriff von Standardsprachlichkeit und wie könnte er gefasst sein? In: Hagemann, Jörg/Klein, Wolf Peter/ Staffeldt, Sven ( $\mathrm{Hg}$ ): Pragmatischer Standard. (= Stauffenburg Linguistik 73). Tübingen, S. 15-33.

Klein, Wolfgang (1986): Der Wahn vom Sprachverfall und andere Mythen. In: Zeitschrift für Literaturwissenschaft und Linguistik 62, S. 11-28.

Lange, Maria B. (2008): Sprachnormen im Spannungsfeld schriftsprachlicher Theorie und Praxis. Die Protokolle der Commerzdeputation Hamburg im 17. Jahrhundert. (= Studia Linguistica Germanica 93). Berlin. 
Langer, Nils (2001): Linguistic purism in action. How auxiliary tun was stigmatized in early New High German. (= Studia Linguistica Germanica 60). Berlin. [Zugl.: Diss., Univ. Newcastle upon Tyne, 2000.]

Law, Claudia (2006): Sprachratgeber und Stillehren in Deutschland (1923-1967). Ein Vergleich der Sprach- und Stilauffassung in vier politischen Systemen. (= Studia Linguistica Germanica 84). Berlin/New York.

Lühr, Rosemarie (1992): Gleichartigkeit, Vollständigkeit, Vermeidung von Redundanz. Prinzipien von Sprachbewertungen im 19. Jahrhundert. In: Muttersprache 102, S. 341-358.

Maercker, Patrizia (2006): Das Sprachverfallsdenken und seine Institutionalisierung. In: Aptum. Zeitschrift für Sprachkritik und Sprachkultur 3, S. 206-237.

Maitz, Péter/Elspaß, Stephan (2007): Warum der „Zwiebelfisch" nicht in den Deutschunterricht gehört. In: Info DaF 34, S. 515-526.

Matthias, Theodor (1892): Sprachleben und Sprachschäden. Ein Führer durch die Schwankungen und Schwierigkeiten des deutschen Sprachgebrauchs. Leipzig.

Meinunger, André (2008): Sick of Sick? Ein Streifzug durch die Sprache als Antwort auf den „Zwiebelfisch“. 2. Aufl. Berlin.

Metzler Lexikon Sprache (2010). Hrsg. v. Helmut Glück. 4., aktual. u. überarb. Aufl. Stuttgart/Weimar.

Meyer, Kerstin (1993): Wustmanns, Sprachdummheitenc. Untersuchungen zu einem Sprachratgeber des 19. Jahrhunderts. In: Sprachwissenschaft 18, S. 223-315.

Polenz, Peter von (1994): Deutsche Sprachgeschichte vom Spätmittelalter bis zur Gegenwart. Bd. 2: 17. und 18. Jahrhundert. Berlin/New York.

Polenz, Peter von (1999): Deutsche Sprachgeschichte vom Spätmittelalter bis zur Gegenwart. Bd. 3: 19. und 20. Jahrhundert. Berlin/New York.

Reichmann, Oskar (1988): Zur Vertikalisierung des Variantenspektrums in der jüngeren Sprachgeschichte des Deutschen. In: Munske, Horst Haider et al. (Hg): Deutscher Wortschatz. Lexikologische Studien. Ludwig Erich Schmitt zum 80. Geburtstag von seinen Marburger Schülern. Berlin/New York, S. 151-180.

Riegel, Mareike (2007): Sprachberatung im Kontext von Sprachpflege und im Verhältnis zu Nachschlagewerken. Unter besonderer Beachtung der Sprachberatungsstelle des Wissen-Media-Verlages. Internet: www.freidok.uni-freiburg.de/volltexte/3593/ (Stand: 12.8.2013).

Sandig, Barbara (1973): Zur Historischen Kontinuität normativ diskriminierter syntaktischer Muster in spontaner Sprechsprache. In: Deutsche Sprache 1, S. 37-57.

Sauter, Anke (2000): Eduard Engel: Literaturhistoriker, Stillehrer, Sprachreiniger. Ein Beitrag zur Geschichte des Purismus in Deutschland. (= Dr. Rabes Doktorhüte 4). Bamberg.

Schlaefer, Michael (1980): Grundzüge der deutschen Orthographiegeschichte vom Jahre 1800 bis zum Jahre 1870. In: Sprachwissenschaft 5, S. 276-319.

Schlaefer, Michael (1981): Der Weg zur deutschen Einheitsorthographie vom Jahre 1870 bis zum Jahre 1901. In: Sprachwissenschaft 6, S. 391-438. 
Schmidt, Wilhelm (2007): Geschichte der deutschen Sprache: Ein Lehrbuch für das germanistische Studium. 10, verb. u. erw. Aufl. Stuttgart.

Schmidt-Wilpert, Gabriele/Lappé, Winfried (1981): Die Entwicklung der Interpunktionsnorm und ihre Kodifizierung im 19. Jahrhundert. In: Zeitschrift für deutsche Philologie 100, S. 390-416.

Schneider, Jan Georg (2011): Was ist richtiges und gutes Deutsch? Sprachratgeber auf dem Prüfstand. In: Arendt, Birte/Kiesendahl, Jana (Hg.): Sprachkritik in der Schule. Theoretische Grundlagen und ihre praktische Relevanz. Göttingen, S. 7389.

Scholze-Stubenrecht, Werner (1995): Bewertungskriterien der Duden-Sprachberatung. In: Biere, Bernd Ulrich/Hoberg, Rudolf (Hg.): Bewertungskriterien in der Sprachberatung. (= Studien zur deutschen Sprache 2). Tübingen, S. 55-61.

Scholze-Stubenrecht, Werner (2002): „Die Auswahl der Einträge ist äußerst beliebig. ${ }^{\text {" }}$ Warum Jagdherr und Pokémon nicht im Duden stehen. In: Sprachwissenschaft 27, S. 225-248.

Schröder, Anne/Busse, Ulrich/Schneider, Ralf (2012): Codification, canons and curricula. Description and prescription in language and literature. (= Bielefelder Schriften zu Linguistik und Literaturwissenschaft 26; The Bielefeld English and American Studies Series in the Bielefelder Schriften zu Linguistik und Literaturwissenschaft 4). Bielefeld.

Stanze, Britta (1994): Die orthographischen Regelbücher des Deutschen. (= Deutsche Hochschulschriften 1044). Egelsbach u.a.

Takada, Hiroyuki (1997): Orthographische Vorschrift und Praxis im Barock. Zum Anteil der Grammatiker an der schriftsprachlichen Norm. In: Zeitschrift für deutsche Philologie 116, S. 68-89.

Takahashi, Hideaki (1996): Die richtige Aussprache des Deutschen in Deutschland, Österreich und der Schweiz nach Massgabe der kodifizierten Normen. (= Duisburger Arbeiten zur Sprach- und Kulturwissenschaft 27). Frankfurt a.M. u.a.

Voeste, Anja (1999): Varianz und Vertikalisierung. Zur Normierung der Adjektivdeklination in der ersten Hälfte des 18. Jahrhunderts. (= Amsterdamer Publikationen zur Sprache und Literatur 140). Amsterdam u.a.

Wahrig (2009): Wahrig. Bd. 5: Richtiges Deutsch leicht gemacht. Hrsg. v. d. WahrigRedaktion. Gütersloh/München. 